\title{
Validation of Ankle Strength Measurements by Means of a Hand-Held Dynamometer in Adult Healthy Subjects
}

\author{
Andrea Ancillao, ${ }^{1}$ Eduardo Palermo, ${ }^{1}$ and Stefano Rossi ${ }^{2}$ \\ ${ }^{1}$ Department of Mechanical and Aerospace Engineering, "Sapienza" University of Rome, Via Eudossiana 18, 00184 Roma, Italy \\ ${ }^{2}$ Department of Economics and Management-Industrial Engineering (DEIM), University of Tuscia, Via del Paradiso 47, \\ 01100 Viterbo, Italy
}

Correspondence should be addressed to Stefano Rossi; stefano.rossi@unitus.it

Received 16 March 2017; Revised 13 June 2017; Accepted 28 June 2017; Published 27 July 2017

Academic Editor: Guiyun Tian

Copyright (C) 2017 Andrea Ancillao et al. This is an open access article distributed under the Creative Commons Attribution License, which permits unrestricted use, distribution, and reproduction in any medium, provided the original work is properly cited.

\begin{abstract}
Uniaxial Hand-Held Dynamometer (HHD) is a low-cost device widely adopted in clinical practice to measure muscle force. HHD measurements depend on operator's ability and joint movements. The aim of the work is to validate the use of a commercial HHD in both dorsiflexion and plantarflexion ankle strength measurements quantifying the effects of HHD misplacements and unwanted foot's movements on the measurements. We used an optoelectronic system and a multicomponent load cell to quantify the sources of error in the manual assessment of the ankle strength due to both the operator's ability to hold still the HHD and the transversal components of the exerted force that are usually neglected in clinical routine. Results showed that foot's movements and angular misplacements of HHD on sagittal and horizontal planes were relevant sources of inaccuracy on the strength assessment. Moreover, ankle dorsiflexion and plantarflexion force measurements presented an inaccuracy less than $2 \%$ and higher than $10 \%$, respectively. In conclusion, the manual use of a uniaxial HHD is not recommended for the assessment of ankle plantarflexion strength; on the contrary, it can be allowed asking the operator to pay strong attention to the HHD positioning in ankle dorsiflexion strength measurements.
\end{abstract}

\section{Introduction}

Measurement of the maximum force that a subject can exert during a volitional contraction is a basic clinical procedure often conducted in clinical and rehabilitation frameworks. It is also referred to as strength assessment [1]. Specifically, this technique enables an easy indirect estimation of joint moment, providing basic information about the healthiness of tendons, ligaments, and joint stability [2]. Furthermore, strength evaluation enables the diagnosis of weakness as a consequence of muscular diseases and allows the quantitative assessment of functional recovery in rehabilitation programs [3-8].

As of today, a widespread and commercially available method to measure muscle strength involves the use of the isokinetic dynamometer [9-12]. This methodology showed a high interrater and intrarater reliability and reproducibility in the measurement of joint forces and torques, on subjects of a wide age range, on both lower and upper limb [5, 9, $13,14]$. However, the isokinetic dynamometer is inherently expensive, cumbersome, and not portable and requires a long patient preparation time.

In clinical environments, simpler and faster methods are often preferred to reduce both patient's discomfort and the examination time. Thus, the most adopted methodology to assess strength involves the Hand-Held Dynamometer (HHD), a low-cost, portable, and easy-to-use device. It consists of a small and portable single-axis dynamometer that can be held in hand by a clinician and applied on some defined landmarks, while asking the patient to exert a force against it $[9,14]$.

Despite its advantages, reports on HHD reproducibility and repeatability were controversial [15-18]. Principal causes of low reliability of HHD based method have been identified in poor operators' training and wrong patient's positioning [7]. In fact, HHD based method relies on operator's strength 
and training to contrast the force exerted by the patient, avoiding misplacements [19].

HHD strength measurements can be performed according to two methods [19]: (i) the "make test," in which the examiner holds the dynamometer stable while the subject exerts a maximal force against it, and (ii) the "break test," in which the examiner overcomes the maximum force exerted by the subject, producing a small limb movement in the opposite direction of patient's force. Both methods were proved reliable and repeatable only if the examiner had enough force to contrast the force exerted by the patient [19]. Other studies provided similar results, by showing that strength measurements performed through HHD are operator-dependent and the "break test" requires a larger force exerted by the examiner $[20,21]$. The influence of the operator was tested by Kim et al. [9] by comparing three setups: (i) with the HHD fixed to the distal tibia by a Velcro strap; (ii) with the HHD held by the operator; and, finally, (iii) with an isokinetic dynamometer, assumed as a reference. They found that fixed and nonfixed methods showed good interrater reliability and the higher reliability was reached in the fixed methods. The HHD held by the operator is the assessment method widely adopted by clinicians as it does not require a complex experimental setup [22].

Though strength can be assessed for all human muscles, a particular clinical relevance is conferred to the strength of lower limb muscles, due to the important role they play in day-living tasks (walking, chair rise, climbing, etc.), which may be compromised by neuromotor pathologies and aging [13]. Among the lower limb joints, ankle deserves a special attention, as dorsi/plantarflexion and inversion/eversion are key movements for balance and general functional ability [23], playing an important role in human gait. In fact, It was observed that ankle kinetics are often affected by neuromotor pathologies and may improve after therapies [24-27].

Several studies have been conducted to assess the validity of HHD measurements of ankle strength. Ankle strength of healthy subjects was measured by means of HHD and then compared to an electromechanical dynamometer, that is, a fixed dynamometer that allowed evaluation of isometric force [18]. Results showed that HHD measurements were poorly correlated to the fixed dynamometer, and statistical differences were found between the two datasets. Researchers attributed the results to low examiner's strength and their inability to position and hold the HHD steady. They concluded that HHD strength measurements of the plantar flexors should not be considered valid [18]. However, these results were in disagreement with the results obtained by Spink et al. [23] that found high reliability of ankle strength measurements by means of HHD in both elder and younger participants concluding that HHD is a valid methodology for the evaluation of ankle strength. Hébert et al. [17] found that among all the lower limb joints ankle plantarflexion and ankle dorsiflexion presented the lowest reliability. Therefore, they recommended further studies in this direction, especially regarding the strength evaluation in children with neuromotor disabilities.

From the previously cited studies, operator's inefficiency to hold the HHD in the right position emerged as the main issue in HHD strength measurements related to the ankle joint. In all the reported studies only a reliability analysis was conducted and, to the best of the authors' knowledge, no studies were performed to identify and quantify the sources of inaccuracy that occur in the assessment of ankle strength by means of a HHD. Therefore detailed studies about the quality of clinical measurements are strongly encouraged [28] with the purpose of establishing reliability, reproducibility, and validity of such measurements [29].

The aim of this study was the validation of the manual use of a commercial HHD, which is a uniaxial load cell, in the plantarflexion and dorsiflexion ankle strength measurements quantifying the effects of HHD misplacements and unwanted foot's movements on the measurements. A validation protocol involving a motion capture system and a multiaxial load cell was exploited to measure actual forces and moments exerted by the subject, the HHD position, and the undesired motion of the patient's foot. The present work took advantage of a measurement protocol previously validated and already applied to the analysis of knee strength measurements $[22,30]$.

\section{Materials and Methods}

2.1. Subjects. Thirty healthy adult subjects $(18 \mathrm{M}, 12 \mathrm{~F}$, age: $26.2 \pm 2.1$ years, height: $173.6 \pm 7.2 \mathrm{~cm}$, and weight: $68.1 \pm$ $8.7 \mathrm{~kg}$ ) were enrolled in the study. Participants had never suffered from any neurological or orthopaedic disorders and had never undergone surgery to the lower limb joints. All the subjects were right-handed even though this was not an inclusion criterion. Measurements were conducted at the MARLab (Movement Analysis and Robotic Laboratory of the Children's Hospital Bambino Gesù).

2.2. Study Approval. This study complied with the principles of the Declaration of Helsinki, and it was approved by the Ethical Committee of the Children's Hospital Bambino Gesù in Rome.

2.3. Experimental Setup. Strength measurements were conducted by means of a six-component load cell, that is, the Gamma F/T Sensor (ATI Industrial Automation, USA). The cell was equipped with a force-transferring aluminium layer and a foam layer on top, designed to increase patient's comfort (Figure 1). The range of measurement of the load cell was $400 \mathrm{~N}$ on the principal axis ( $z$-axis), $130 \mathrm{~N}$ on the transversal axes, and $10 \mathrm{Nm}$ for the moment on each axis. Resolution was $1 / 20 \mathrm{~N}$ for the force and $1 / 800 \mathrm{Nm}$ for the moment. Weight of the load cell was $0.255 \mathrm{~kg}$, diameter $75.4 \mathrm{~mm}$, and height $33.3 \mathrm{~mm}$. In this study, we used the above-described load cell as a Hand-Held Dynamometer, named HHD in the following.

Motion and displacements were recorded by means of an 8-camera Vicon MX Optoelectronic System (Oxford Metrics, UK), named OS in the following. Sampling frequency was set at $200 \mathrm{~Hz}$. We used Vicon Nexus 1.7 software (Oxford Metrics, UK) to reconstruct markers' trajectories. System calibration was performed before each acquisition session, according to manufacturer's instruction. The overall RMS error of marker reconstruction in three-dimensional space was $\sim 1 \mathrm{~mm}$ in a calibrated volume of about $3 \mathrm{~m} \times 1 \mathrm{~m} \times 2 \mathrm{~m}$. 


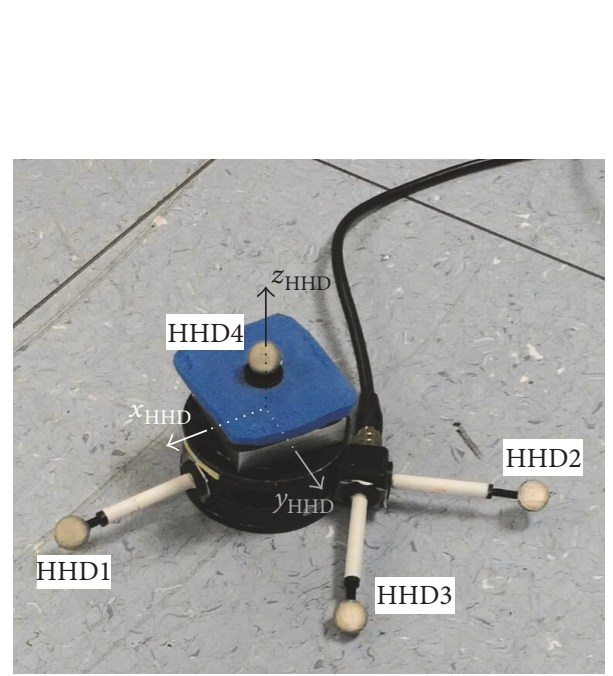

(a)

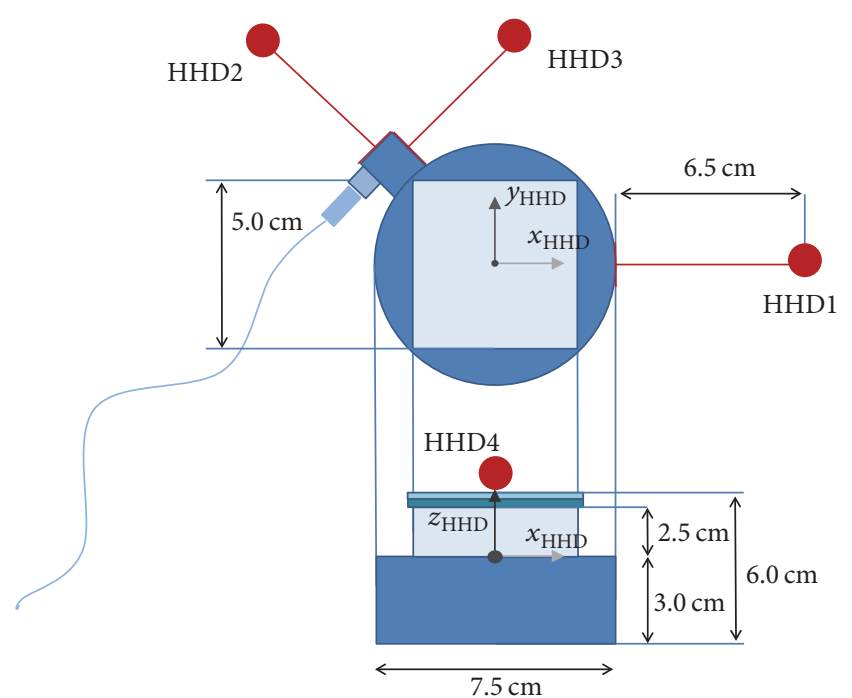

(b)

FIGURE 1: (a) The six-component HHD equipped with motion capture markers. (b) Schematics of the HHD, equipped with the contact part, markers (in red), and the representation of local reference system.

The output signals of the HHD were collected through the analog input ports of the OS ensuring the synchronization between devices.

2.4. Motion Capture Protocol. For this study, we adapted to the ankle joint a protocol previously designed for the knee [30].

Four markers were placed on the HHD (Figures 1(a) and 1(b)). Rigid sticks were used to avoid covering of the markers by the operator's hand. The central marker was placed in the midpoint of the patient-interface area of the HHD. That marker was needed to locate surface center with respect to the other markers. Fourteen markers were placed on the subject's lower limbs (Figure 2). Landmarks were identified as follows: lateral and medial femoral epicondyles (4 markers), lateral and medial malleoli (4 markers), lateral shanks (2 markers), head of first metatarsal (2 markers), and head of fifth metatarsal (2 markers).

A static trial was recorded before the measuring session to identify the reference systems (Figure 1) and to measure the offset signals of the HHD. In the static trial, the HHD was placed on the floor with no load applied on it and the subject was still in a stand-up position. During the measuring session, the central marker was removed and its position was reconstructed by using a localization procedure based on the three fixed markers [31]. We included the left leg in the protocol design, to allow processing of strength trials in lefthanded subject.

2.5. Strength Protocol. Strength of the ankle dorsiflexor and plantarflexor muscles has been measured by applying a validated clinical protocol [32], consisting in a "make test" method $[19,21]$. In both ankle plantarflexion and dorsiflexion movements, the subject was lying on the bed with ankle in neutral position (Figure 2). HHD was placed under the foot sole on the metatarsal region for plantarflexion testing and on the upper metatarsal region for the dorsiflexion one. The subjects were instructed to push against the HHD exerting their maximum force. Strength was measured by a trained clinician (male, height $170 \mathrm{~cm}$, weight $73 \mathrm{~kg}$ ) with a longterm experience in strength assessment. The operator was standing at the bottom of the bed, holding the HHD with both hands in order to keep it in place while he counteracted the patient's force to keep the foot still for about five seconds. The participants were instructed to avoid explosive contraction but to gradually increase force from zero to the maximum achievable value [33].

Trials were repeated five times for both plantarflexion and dorsiflexion with a resting time of about $30 \mathrm{~s}$ between trials to avoid fatigue effects in both subject and operator.

2.6. Data Analysis. Before the identification of local reference frames, we defined the knee and ankle centers as the midpoint between the two markers on epicondyles and malleoli, respectively.

The LRS for the HHD (namely, $\mathrm{LRS}_{\mathrm{HHD}}$ ) is shown in Figure 1(b) and was defined as follows:

(i) vmkrl: virtual marker as the projection of HHD4 on the plane defined by HHD1, HHD2, and HHD3

(ii) $x_{\mathrm{HHD}}$ : unit vector from vmkr1 to HHD1

(iii) $z_{\mathrm{HHD}}$ : unit vector perpendicular to the plane defined by HHD1, HHD2, and HHD3, pointing outwards

(iv) $y_{\mathrm{HHD}}$ : defined as cross product between $z$-and $x$-axes

(v) Origin: virtual marker on the line between vmkr1 and HHD4 with an offset from HHD4 equal to the thickness of the force coupling layers.

The LRS $_{\mathrm{HHD}}$ was designed in such a way that $x$-axis, $y$-axis, and $z$-axis were directed as the respective internal axes of the load cell. 


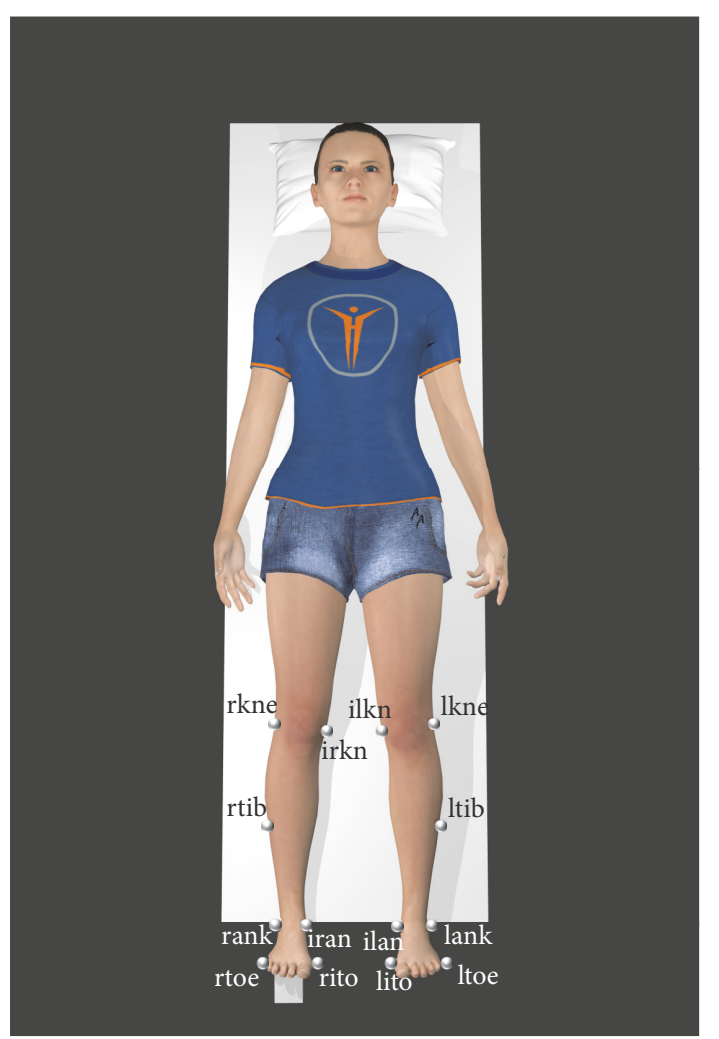

FIGURE 2: Graphical representation of the subject lying on the bed in measurement position wearing the marker protocol used for the trials. The white cube underneath the right foot represents the HHD position. lows:

The LRS for the foot (namely, $\mathrm{LRS}_{\mathrm{FT}}$ ) was defined as fol-

(i) $y_{\mathrm{FT}}$ : unit vector from ankle center to knee center

(ii) $z_{\mathrm{FT}}$ : unit vector perpendicular to the plane defined by the knee center, the ankle center, and midpoint between markers on first and fifth metatarsal, pointing to lateral direction

(iii) $x_{\mathrm{FT}}$ : defined as cross product between $y_{\mathrm{FT}}{ }^{-}$and $z_{\mathrm{FT}^{-}}$ axes

(iv) Origin: ankle center.

The designed setup allowed estimating the following kinematic parameters (Figure 3):

(i) The Range of Motion (RoM) of the ankle dorsi/plantarflexion angle, defined as the difference between the maximum and minimum of angle measured throughout the trial: Ankle angle was computed on the basis of a three-point procedure between knee center, ankle center, and the midpoint between markers on the first and fifth metatarsal. As the ankle should ideally remain still during the strength measurement, RoM was assumed as a quality indicator of strength measurements: a lower RoM indicates a higher quality of the performed measurement.

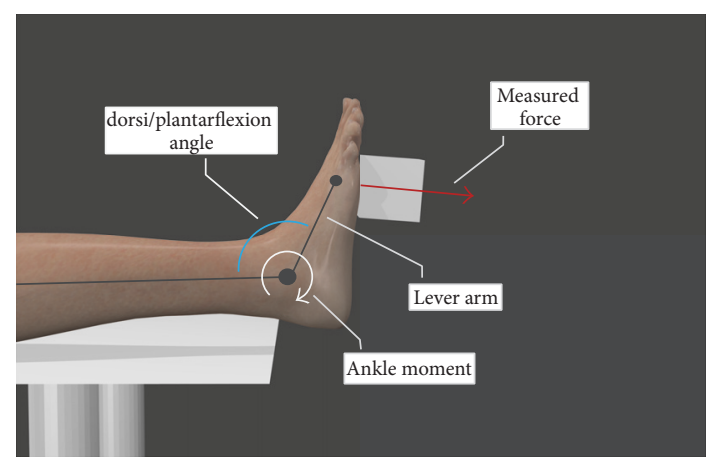

Figure 3: Parameters computed for the ankle strength assessment, lateral view.

(ii) The angles between the HHD $z$-axis and the transverse and sagittal planes of the foot, namely, $A_{1}$ and $A_{2}: A_{1}$ and $A_{2}$ were evaluated when the maximum force from $\mathrm{HHD}$ was recorded. Their deviations, that is, $\delta A_{1}$ and $\delta A_{2}$, from their ideal values $\left(90^{\circ}\right)$ indicate wrong positioning of HHD during the strength measurement. In the ideal case $\delta A_{1}=\delta A_{2}=0^{\circ}$.

The kinematic parameters were computed for both ankle plantarflexion and dorsiflexion trials and then averaged between the five repetitions of each subject. To assess repeatability of measurements, we also computed the Coefficient of Variation (CV) for each parameter. The CV was defined as the percentage ratio between standard deviation (SD) and mean between the five repetitions of each subject.

Kinetic analysis was conducted in terms of forces and moments acting on the ankle joint. Forces and moments were expressed in the LRS of the foot $\left({ }^{\mathrm{FT}} \mathbf{F}\right.$ and $\left.{ }^{\mathrm{FT}} \mathbf{M}\right)$ :

$$
\begin{aligned}
{ }^{\mathrm{FT}} \mathbf{F} & ={ }^{\mathrm{FT}} \mathbf{R}_{\mathrm{HHD}} \cdot{ }^{\mathrm{HHD}} \mathbf{F}, \\
{ }^{\mathrm{FT}} \mathbf{M} & ={ }^{\mathrm{FT}} \mathbf{R}_{\mathrm{HHD}} \cdot{ }^{\mathrm{HHD}} \mathbf{M}+{ }^{\mathrm{FT}} \mathbf{o}_{\mathrm{HHD}} \times{ }^{\mathrm{FT}} \mathbf{F},
\end{aligned}
$$

where ${ }^{\mathrm{HHD}} \mathbf{F}$ and ${ }^{\mathrm{HHD}} \mathbf{M}$ are the outputs of the HHD, ${ }^{\mathrm{FT}} \mathbf{R}_{\mathrm{HHD}}$ is the rotational matrix between $\mathrm{LRS}_{\mathrm{HHD}}$ and $\mathrm{LRS}_{\mathrm{FT}}$, and ${ }^{\mathrm{FT}} \mathbf{o}_{\mathrm{HHD}}$ is the origin of $\mathrm{LRS}_{\mathrm{HHD}}$ expressed in $\mathrm{LRS}_{\mathrm{FT}}$.

From ${ }^{\mathrm{FT}} \mathbf{F}$ and ${ }^{\mathrm{FT}} \mathbf{M}$, we defined

(i) $F_{M}$, as the maximum value of ${ }^{\mathrm{FT}} F_{y}$, which represents the measure of the strength;

(ii) $F_{T}$, as the transverse component of the force exerted by the subject (it represents the intensity of lateral forces that cannot be gathered by means of a singlecomponent load cell):

$$
F_{T}=\sqrt{{ }^{\mathrm{FT}} F_{x}{ }^{2}+{ }^{\mathrm{FT}} F_{z}{ }^{2}}
$$

(iii) $M_{M}$, as the maximum value of ${ }^{\mathrm{FT}} M_{z}$, which represents the ankle dorsi/plantarflexion moment when the strength measure is performed;

(iv) $M_{T}$, as the transverse component of the knee moment:

$$
M_{T}=\sqrt{{ }^{\mathrm{FT}} M_{x}{ }^{2}+{ }^{\mathrm{FT}} M_{y}{ }^{2}} .
$$


All these parameters were averaged across the five repetitions for each subject. As for the kinematic parameters, we computed the Coefficient of Variation (CV) for all the kinetic parameters, to assess the repeatability of the procedure.

In order to simulate the strength measurements that are usually gathered in clinical routine by using a uniaxial $\mathrm{HHD}$, we simulated its output calculating the above-reported parameters considering only the force measured on the $z$ axis of the HHD and placing the other force components and the moments equal to zero. The maximum value of force was assumed as the nominal strength measurement $(\widehat{F})$ that is the only one that can be measured in clinical routine (see (4)). The respective nominal knee moment $(\widehat{M})$ was estimated by multiplying $\widehat{F}$ by the lever arm $(d)$ between the center of HHD and the ankle joint; $d$ was measured with a tape measure as made in clinical routine (see (5))

$$
\begin{aligned}
\widehat{F} & =\max \left({ }^{\mathrm{HHD}} F_{z}\right), \\
\widehat{M} & =d \cdot \widehat{F} .
\end{aligned}
$$

The differences between the nominal $\widehat{F}, \widehat{M}$ and the respective reference values obtained using the proposed validation procedure $\left(F_{M}\right.$ and $\left.M_{M}\right)$ were quantified in terms of Root Mean Square Error (RMSE):

$$
\begin{aligned}
\operatorname{RMSE}_{F} & =\sqrt{\frac{\sum_{i=1}^{N}\left(F_{M}^{i}-\widehat{F}_{i}\right)^{2}}{N}} \cdot \frac{100}{\max _{i}\left(\widehat{F}_{i}\right)}[\%], \\
\operatorname{RMSE}_{M} & =\sqrt{\frac{\sum_{i=1}^{N}\left(M_{M}^{i}-\widehat{M}_{i}\right)^{2}}{N}} \cdot \frac{100}{\max _{i}\left(\widehat{M}_{i}\right)}[\%] .
\end{aligned}
$$

$\mathrm{RMSE}_{F}$ and $\mathrm{RMSE}_{M}$ allowed the quantification of the accuracy of uniaxial HHD in the estimation of ankle strength and moment measurements, respectively.

Finally, we calculated also the index $Q_{\text {index }}$ to provide an overall quantification of the quality of the strength measurements [30]. Specifically, $Q_{\text {index }}($ see (7)) takes into account both the angular displacement of HHD and the transverse component of moment. The higher the value of $Q_{\text {index }}$ is, the higher the quality of strength measurement is. Its ideal value is $100 \%$

$$
\begin{aligned}
& Q_{\text {index }} \\
& \quad=100\left(1-\sqrt{\left(\frac{\delta A_{1}}{90}\right)^{2}+\left(\frac{\delta A_{2}}{90}\right)^{2}+\left(\frac{M_{T}}{M_{M}}\right)^{2}}\right) .
\end{aligned}
$$

The identification of local reference systems (LRS) for body segments and HHD and the estimation of kinematic and kinetic parameters were implemented by means of Matlab (MathWorks, USA).

2.7. Statistics. Repeatability of the measured parameters was assessed by computing CVs while RMSE parameters allowed quantifying the inaccuracy occurring when lateral components of force and moment are neglected, that is, when a commercial uniaxial HHD is used. All data were tested for normality by means of the Shapiro-Wilk test. Since data were proved to be normally distributed, the $t$-test was used to assess differences between means. Tests were assumed significant if $p$ was lower than 0.05. Moreover, in order to analyze the influence of an unwanted displacement of HHD on the accuracy of the HHD measurements and on the quality of strength measurements, the Pearson productmoment correlation coefficient $r$ was computed to study the correlation between kinematic and kinetic parameters. A strong correlation was assumed if $|r|$ was higher than 0.7.

\section{Results and Discussion}

Means and standard deviations of both kinematic and kinetic parameters and $p$ values are reported in Table 1 . The observed RoMs were not equal to $0^{\circ}$, indicating that the ankle was moving during the measurements. Therefore the operator was not able to keep the HHD and the foot completely still with an undesired motion of the foot during the trial. This finding was in line with the results of Kim et al. [9] that demonstrated a decreased measurement validity when the dynamometer is not fixed but held in hand by the operator. Moreover, the observed RoM was slightly higher for plantarflexion trials $(p=0.06)$, where a higher exerted force was registered implying more difficulty in keeping the foot still when a high level of force occurred.

Angular displacements $\delta A_{1}$ and $\delta A_{2}$ were higher in plantarflexion trials than dorsiflexion ones (Table 1). This could be due to the higher force exerted in plantarflexion trials; in fact it reduced operator's ability to keep the HHD in place during the measurement. On the contrary, the operator was able to maintain still the HHD during dorsiflexion trials since angular displacements were low. Consequently, the angular misplacements of HHD on sagittal and horizontal planes are relevant sources of inaccuracy mainly in the plantarflexion strength assessment. Comparing the kinetic parameters between the two directions, the plantarflexion trials showed higher differences between the actual and the measurement forces and moments than those in dorsiflexion. The lateral components of force and moment $F_{T}$ and $M_{T}$ were both higher for plantarflexion than for dorsiflexion; it could be due to a wrong angular positioning of the HHD on both planes, as observed by means of the $\delta A_{1}$ and $\delta A_{2}$ coefficients that were higher in plantarflexion (Table 1). The kinematic and kinetic analysis suggested a higher validity of ankle dorsiflexion trials than the plantarflexion ones.

As regards the accuracy of ankle strength measurements, we observed that $\widehat{F}$ and $\widehat{M}$ were higher than $F_{M}$ and $M_{M}$ for both directions, while the transversal components $F_{T}$ and $M_{T}$ were not negligible. $\widehat{F}$ and $\widehat{M}$ represented the force and moment commonly measured by means of clinical HHD and $F_{M}$ and $M_{M}$ were the actual values. In case of misplacement, the force and moment measured by a commercial HHD differ significantly from the force and moment effectively exerted by the joint. Our findings implied that wrong positioning of HHD increased the lateral components of force reducing the force on the main axis. As regards the analysis of RMSE, we found very low value of $\mathrm{RMSE}_{F}$ in dorsiflexion $(<5 \%)$, 
TABLE 1: Mean (SD) values of parameters measured for the ankle plantarflexion and dorsiflexion. The $p$ values are reported in the last column. * indicates a significant difference $(p<0.05)$.

\begin{tabular}{lccc}
\hline & Plantarflexion & Dorsiflexion & $p$ \\
\hline RoM [ $\left.{ }^{\circ}\right]$ & $26.7(9.9)$ & $21.1(6.1)$ & 0.06 \\
$\delta A_{1}\left[^{\circ}\right]$ & $29.5(8.7)$ & $5.1(2.9)$ & $<0.01^{*}$ \\
$\delta A_{2}\left[^{\circ}\right]$ & $12.9(5.4)$ & $5.1(3.3)$ & $<0.01^{*}$ \\
$\widehat{F}[\mathrm{~N}]$ & $244.3(46.2)$ & $191.8(38.5)$ & $<0.01^{*}$ \\
$\widehat{M}[\mathrm{Nm}]$ & $54.0(15.7)$ & $36.5(10.1)$ & $<0.01^{*}$ \\
$F_{M}[\mathrm{~N}]$ & $209.7(39.4)$ & $189.8(39.2)$ & 0.14 \\
$F_{T}[\mathrm{~N}]$ & $125.0(31.2)$ & $34.3(13.9)$ & $<0.01^{*}$ \\
$M_{M}[\mathrm{Nm}]$ & $30.5(4.8)$ & $23.7(4.9)$ & $<0.01^{*}$ \\
$M_{T}[\mathrm{Nm}]$ & $12.9(4.4)$ & $3.0(1.3)$ & $<0.01^{*}$ \\
$\mathrm{RMSE}_{F}[\%]$ & $13.3(5.4)$ & $1.6(1.3)$ & $<0.01^{*}$ \\
$\mathrm{RMSE}_{M}[\%]$ & $35.3(11.1)$ & $29.4(11.8)$ & 0.14 \\
$\mathrm{CV}_{\mathrm{RoM}}[\%]$ & $17.6(10.0)$ & $21.1(9.9)$ & \\
$\mathrm{CV}_{\delta A 1}[\%]$ & $16.2(10.0)$ & $50.8(29.1)$ & $<.31$ \\
$\mathrm{CV}_{\delta A 2}[\%]$ & $26.1(19.1)$ & $49.1(25.2)$ & $<0.01^{*}$ \\
$\mathrm{CV}_{\widehat{F}}[\%]$ & $7.2(5.1)$ & $8.1(4.4)$ & $<0.01^{*}$ \\
$\mathrm{CV}_{\widehat{M}}[\%]$ & $16.7(12.1)$ & $15.6(7.1)$ & 0.57 \\
$Q_{\text {index }}[\%]$ & $44.2(11.0)$ & $88.2(5.0)$ & $<0.75$ \\
\hline
\end{tabular}

while it was higher for plantarflexion $(<15 \%)$ confirming both that the ankle strength assessments were more accurate when low force values occurred and that the analysis of plantar-flexor strength may be more difficult to be performed by clinicians. These findings were confirmed by the higher lateral components of the force exerted by the ankle in the plantarflexion movement.

As regards the repeatability of ankle strength measurements, CVs were computed to quantify the variability within the same subject. The highest values of CVs were observed for $\mathrm{CV}_{\delta A 1}$ and $\mathrm{CV}_{\delta A 2}$ during dorsiflexion trials ( 50\%). This result proved a poor repeatability in terms of HHD positioning also when the operator was able to maintain still the HHD, demonstrating that the strength measurements are likely influenced by the strength of the examiner, in accordance with the findings of other studies $[17,18]$. Average values of $\mathrm{CV}_{\widehat{F}}$ were less than $10 \%$ and average values of $\mathrm{CV}_{\widehat{M}}$ were less than $20 \%$ for both plantarflexion and dorsiflexion, indicating a good intrasubject repeatability of the force measurement. The repeatability of moments was lower than the force. This finding is likely due to the wrong positioning of the HDD since an increase of variability could be due to a wrong estimation of the lever arm, that is, the distance between the HHD position and the ankle center. No statistical differences were observed between plantarflexion and dorsiflexion.

Finally, we computed a synthetic index, $Q_{\text {index }}$, representing the overall quality of the measurement (Table 1). It was conceived to account for both the angular misplacements of the HHD and the undesired lateral components of moment. Its average value resulted lower for plantarflexion than dorsiflexion. It was in accordance with the other parameters that identified the most relevant inaccuracies in the ankle plantarflexion trials. This finding was in agreement with other works that reported a poor repeatability and reliability of ankle strength measurements, especially for plantarflexion trials $[17,18]$. From a comparison of the $Q_{\text {index }}$ values with the ones evaluated for the knee strength measurements [30], it emerges that, among the strength measurements, the plantarflexion analysis is the more complex one to be performed and it implicates low values of accuracy in force and moment measurements and a low ability of the operator to maintain still the HHD. On the contrary, the quality of ankle dorsiflexion strength measurements is comparable with the knee flexion and extension ones.

Correlation analyses between kinematic and kinetic parameters were performed to analyze the influence of an unwanted displacement of HHD on the accuracy of the HHD measurements (Table 2). A strong correlation was found only between the RoM and $\mathrm{RMSE}_{M}$ indicating that the intensity of the undesired movement of the foot had effect on the measured moment. The accuracy of HHD in the moment measurements was not strongly related to a wrong orientation of the load cell but it depends mainly on the unwanted movement of the foot during the experimental trial.

In conclusion, ankle strength assessment by means of a commercial uniaxial HHD can be considered consistent for dorsiflexion trials, as $F_{T}, M_{T}$, and $\mathrm{RMSE}_{F}$ measured in this study were relatively low. Differences between $\widehat{F}$ and $F_{M}$ were low and the average quality index was relatively high. Thus, the estimated inaccuracy could be considered acceptable for the clinical use of uniaxial HHDs. However, it is always recommended to pay attention to HHD positioning. Conversely, plantarflexion trials involved higher exerted force and implied a lower value of the quality index to which higher 
TABLE 2: Correlation coefficients $(r)$ between kinematic indices and kinetic indices for the ankle plantarflexion and dorsiflexion. $*$ indicates a strong correlation $(|r| \geq 0.7)$.

\begin{tabular}{lccccc}
\hline & & $F_{T}$ & $M_{T}$ & RMSE $_{F}$ & RMSE $_{M}$ \\
\hline \multirow{3}{*}{ Ankle plantarflexion } & $\mathrm{RoM}$ & 0.1 & 0.3 & 0.0 & -0.3 \\
& $\delta A_{1}$ & -0.1 & -0.5 & 0.3 & -0.1 \\
& $\delta A_{2}$ & 0.3 & 0.3 & 0.1 & 0.0 \\
\hline \multirow{3}{*}{ Ankle dorsiflexion } & $\mathrm{RoM}$ & -0.2 & -0.1 & -0.3 & $0.7^{*}$ \\
& $\delta A_{1}$ & 0.1 & -0.4 & 0.3 & 0.0 \\
& $\delta A_{2}$ & 0.4 & 0.4 & 0.4 & -0.3 \\
\hline
\end{tabular}

RMSE values and higher intensity of lateral components of force and moment corresponded. Inherent validity of HHD measurements of plantarflexion strength is consequently low.

3.1. Study Limitations. The main limitations of the work are that only one operator performed the experimental trials and that we analyzed only adult healthy subjects. Since the aim of the study was not the quantification of the ability of operators in performing the ankle strength measurements but it was the analysis of the effects of unwanted HHD displacements on strength measurements, we decided to use only one operator in order to avoid possible confounding effects. Moreover we decided to analyze only adult healthy subjects since they were assumed as the worst-case scenario. In fact, in children and adults with pathology related to the generation of muscle force, the exerted forces are lower than the ones generated by healthy adults and, therefore, lower measurement inaccuracies related to the displacements of HHD should be observed. Further studies involving both the analysis of interoperator reproducibility by comparing the analyzed parameters gathered by operators with different level of ability and the validation of HHD strength measurements in pediatric and patient populations may be performed.

\section{Conclusions}

This work validated the use of a commercial HHD in both dorsiflexion and plantarflexion ankle strength measurements quantifying the effects of HHD misplacements and unwanted foot's movements on the measurements performed by an expert and trained clinician. The foot's movements and angular misplacements of HHD on sagittal and horizontal planes were identified as relevant sources of inaccuracy of the strength assessment. The dorsiflexion trials could be considered more reliable than the plantarflexion ones, which showed higher errors and lower values of the quality index. In conclusion, commercial uniaxial HHDs are not recommended for the assessment of ankle plantarflexion strength but they should be used carefully in the estimation of the ankle dorsiflexion strength. Clinical protocols should be revised in order to ensure proper limb fixation and to reduce both the effects of foot motion and the HHD positioning errors on the strength measurements.

\section{Conflicts of Interest}

The authors declare that they have no conflicts of interest.

\section{Acknowledgments}

This research was supported by the Seventh Framework Program FP7 (MD-Paedigree, FP7-ICT-2011-9; co-PI: P. Cappa). The authors would like to acknowledge Professor Paolo Cappa, who passed away on August 26, 2016. His contribution was fundamental to planning the work. His tireless effort along with his precious suggestions led the team to a remarkable professional growth. The authors wish to acknowledge the M.S. students of "Sapienza" University of Rome that voluntarily took part in this study and the clinical staff of Children's Hospital "Bambino Gesù" of Rome for the use of their lab and for the help in data acquisition.

\section{References}

[1] E. T. Berry, C. A. Giuliani, and D. L. Damiano, "Intrasession and intersession reliability of handheld dynamometry in children with cerebral palsy," Pediatric Physical Therapy, vol. 16, no. 4, pp. 191-198, 2004.

[2] R. Brunner and E. Rutz, "Biomechanics and muscle function during gait," Journal of Children's Orthopaedics, vol. 7, no. 5, pp. 367-371, 2013.

[3] R. W. Bohannon, "Hand-held compared with isokinetic dynamometry for measurement of static knee extension torque (parallel reliability of dynamometers)," Clinical Physics and Physiological Measurement, vol. 11, no. 3, pp. 217-222, 1990.

[4] G. M. Allen, S. C. Gandevia, and D. K. McKenzie, "Reliability of measurements of muscle strength and voluntary activation using twitch interpolation," Muscle \& Nerve, vol. 18, no. 6, pp. 593-600, 1995.

[5] V. A. Hughes, W. R. Frontera, M. Wood et al., "Longitudinal muscle strength changes in older adults: influence of muscle mass, physical activity, and health," The Journals of Gerontology A: Biological Sciences and Medical Sciences, vol. 56, no. 5, pp. B209-B217, 2001.

[6] R. J. Maughan, J. S. Watson, and J. Weir, "Strength and cross-sectional area of human skeletal muscle," The Journal of Physiology, vol. 338, no. 1, pp. 37-49, 1983.

[7] S. Bandinelli, E. Benvenuti, I. Del Lungo et al., "Measuring muscular strength of the lower limbs by hand-held dynamometer: a standard protocol," Aging Clinical and Experimental Research, vol. 11, no. 5, pp. 287-293, 1999.

[8] A. Ancillao, M. Galli, C. Rigoldi, and G. Albertini, "Linear correlation between fractal dimension of surface EMG signal from Rectus Femoris and height of vertical jump," Chaos, Solitons and Fractals, vol. 66, pp. 120-126, 2014. 
[9] W. K. Kim, D.-K. Kim, K. M. Seo, and S. H. Kang, "Reliability and validity of isometric knee extensor strength test with handheld dynamometer depending on its fixation: a pilot study," Annals of Rehabilitation Medicine, vol. 38, no. 1, pp. 84-93, 2014.

[10] D. E. Tsaopoulos, V. Baltzopoulos, P. J. Richards, and C. N. Maganaris, "Mechanical correction of dynamometer moment for the effects of segment motion during isometric kneeextension tests," Journal of Applied Physiology, vol. 111, no. 1, pp. 68-74, 2011.

[11] H. J. Martin, V. Yule, H. E. Syddall, E. M. Dennison, C. Cooper, and A. A. Sayer, "Is hand-held dynamometry useful for the measurement of quadriceps strength in older people? a comparison with the gold standard biodex dynamometry," Gerontology, vol. 52, no. 3, pp. 154-159, 2006.

[12] J. C. Janssen and L. Le-Ngoc, "Intratester reliability and validity of concentric measurements using a new hand-held dynamometer," Archives of Physical Medicine and Rehabilitation, vol. 90, no. 9, pp. 1541-1547, 2009.

[13] A. Hartmann, R. Knols, K. Murer, and E. D. De Bruin, "Reproducibility of an isokinetic strength-testing protocol of the knee and ankle in older adults," Gerontology, vol. 55, no. 3, pp. 259-268, 2009.

[14] M. L. Fulcher, C. M. Hanna, and C. Raina Elley, "Reliability of handheld dynamometry in assessment of hip strength in adult male football players," Journal of Science and Medicine in Sport, vol. 13, no. 1, pp. 80-84, 2010.

[15] R. W. Bohannon and A. W. Andrews, "Interrater reliability of hand-held dynamometry," Physical Therapy, vol. 67, no. 6, pp. 931-933, 1987.

[16] D. L. Riddle, S. D. Finucane, J. M. Rothstein, and M. L. Walker, "Intrasession and intersession reliability of hand-held dynamometer measurements taken on brain-damaged patients," Physical Therapy, vol. 69, no. 3, pp. 182-189, 1989.

[17] L. J. Hébert, D. B. Maltais, C. Lepage, J. Saulnier, M. Crête, and M. Perron, "Isometric muscle strength in youth assessed by hand-held dynamometry: a feasibility, reliability, and validity study," Pediatric Physical Therapy, vol. 23, no. 3, pp. 289-299, 2011.

[18] A. R. Marmon, F. Pozzi, A. H. Alnahdi, and J. A. Zeni, “The validity of plantarflexor strength measures obtained through hand-held dynamometry measurements of force," International Journal of Sports Physical Therapy, vol. 8, no. 6, pp. 820-827, 2013.

[19] R. W. Bohannon, "Make tests and break tests of elbow flexor muscle strength," Physical Therapy, vol. 68, no. 2, pp. 193-194, 1988.

[20] B. A. Phillips, S. K. Lo, and F. L. Mastaglia, "Muscle force measured using 'break' testing with a hand-held myometer in normal subjects aged 20 to 69 years," Archives of Physical Medicine and Rehabilitation, vol. 81, no. 5, pp. 653-661, 2000.

[21] B. A. Laing, F. L. Mastaglia, S. K. Lo, and P. Zilko, "Comparative assessment of knee strength using hand-held myometry and isometric dynamometry in patients with inflammatory myopathy," Physiotherapy Theory and Practice, vol. 11, no. 3, pp. 151-156, 1995.

[22] A. Ancillao, S. Rossi, F. Patane, and P. Cappa, "A preliminary study on quality of knee strength measurements by means of hand held dynamometer and optoelectronic system," in Proceedings of the 2015 IEEE International Symposium on Medical Measurements and Applications (MeMeA '15), pp. 595-599, Turin, Italy, May 2015.
[23] M. J. Spink, M. R. Fotoohabadi, E. Wee, K. D. Hill, S. R. Lord, and H. B. Menz, "Foot and ankle strength, range of motion, posture, and deformity are associated with balance and functional ability in older adults," Archives of Physical Medicine and Rehabilitation, vol. 92, no. 1, pp. 68-75, 2011.

[24] M. Galli, C. Rigoldi, R. Brunner, N. Virji-Babul, and A. Giorgio, "Joint stiffness and gait pattern evaluation in children with Down syndrome," Gait and Posture, vol. 28, no. 3, pp. 502-506, 2008.

[25] C. Rigoldi, M. Galli, V. Cimolin et al., "Gait strategy in patients with Ehlers-Danlos syndrome hypermobility type and Down syndrome," Research in Developmental Disabilities, vol. 33, no. 5, pp. 1437-1442, 2012.

[26] V. Cimolin, F. Camerota, C. Celletti et al., "The effects of neuromuscular taping on gait walking strategy in a patient with joint hypermobility syndrome/Ehlers-Danlos syndrome hypermobility type," Therapeutic Advances in Musculoskeletal Disease, vol. 7, no. 1, pp. 3-10, 2015.

[27] L. Vismara, V. Cimolin, M. Galli, G. Grugni, A. Ancillao, and P. Capodaglio, "Osteopathic Manipulative Treatment improves gait pattern and posture in adult patients with Prader-Willi syndrome," International Journal of Osteopathic Medicine, vol. 19, pp. 35-43, 2016.

[28] L. B. Mokkink, C. B. Terwee, D. L. Knol et al., "The COSMIN checklist for evaluating the methodological quality of studies on measurement properties: a clarification of its content," BMC Medical Research Methodology, vol. 10, article 22, 2010.

[29] C. B. Terwee, S. D. M. Bot, M. R. de Boer et al., "Quality criteria were proposed for measurement properties of health status questionnaires," Journal of Clinical Epidemiology, vol. 60, no. 1, pp. 34-42, 2007.

[30] A. Ancillao, S. Rossi, and P. Cappa, "Analysis of knee strength measurements performed by a hand-held multicomponent dynamometer and optoelectronic system," IEEE Transactions on Instrumentation and Measurement, vol. 66, no. 1, pp. 85-92, 2017.

[31] A. Ancillao, M. Galli, S. L. Vimercati, and G. Albertini, "An optoelectronic based approach for handwriting capture," Computer Methods and Programs in Biomedicine, vol. 111, no. 2, pp. 357-365, 2013.

[32] M. N. Eek, A.-K. Kroksmark, and E. Beckung, "Isometric muscle torque in children 5 to 15 years of age: normative data," Archives of Physical Medicine and Rehabilitation, vol. 87, no. 8, pp. 1091-1099, 2006.

[33] Y.-P. Wuang, J.-J. Chang, M.-H. Wang, and H.-C. Lin, "Testretest reliabilities of hand-held dynamometer for lower-limb muscle strength in intellectual disabilities," Research in Developmental Disabilities, vol. 34, no. 8, pp. 2281-2290, 2013. 


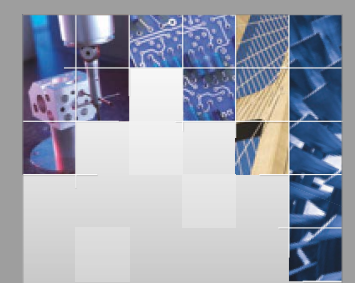

\section{Enfincering}
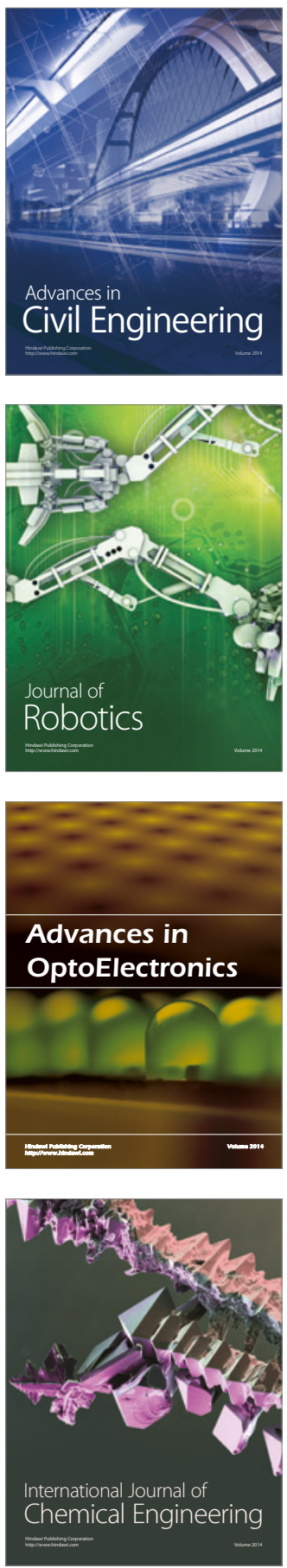

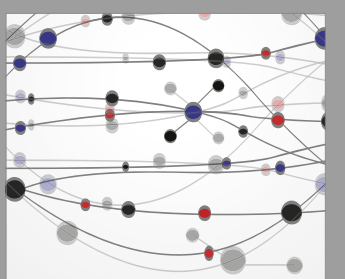

The Scientific World Journal

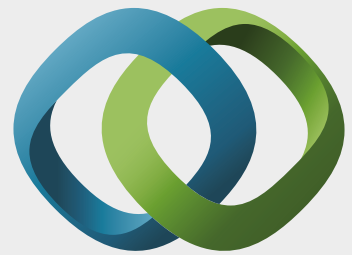

\section{Hindawi}

Submit your manuscripts at

https://www.hindawi.com
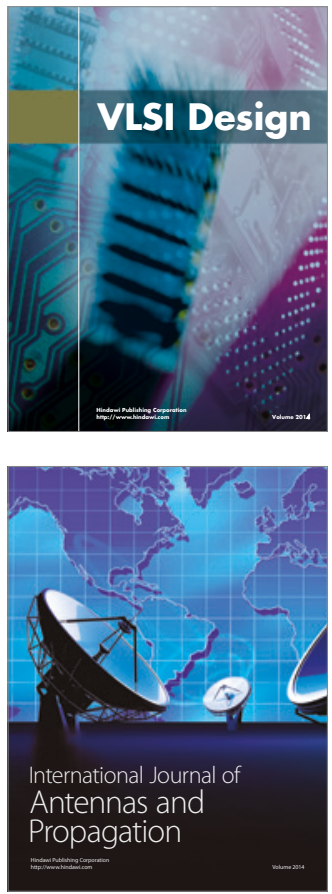

\section{Rotating}

Machinery
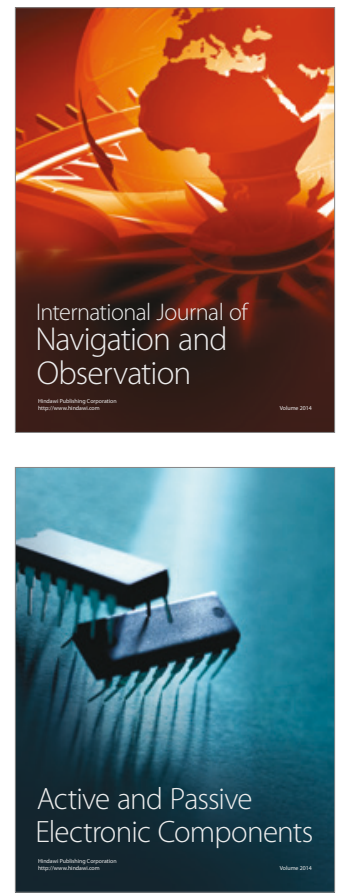
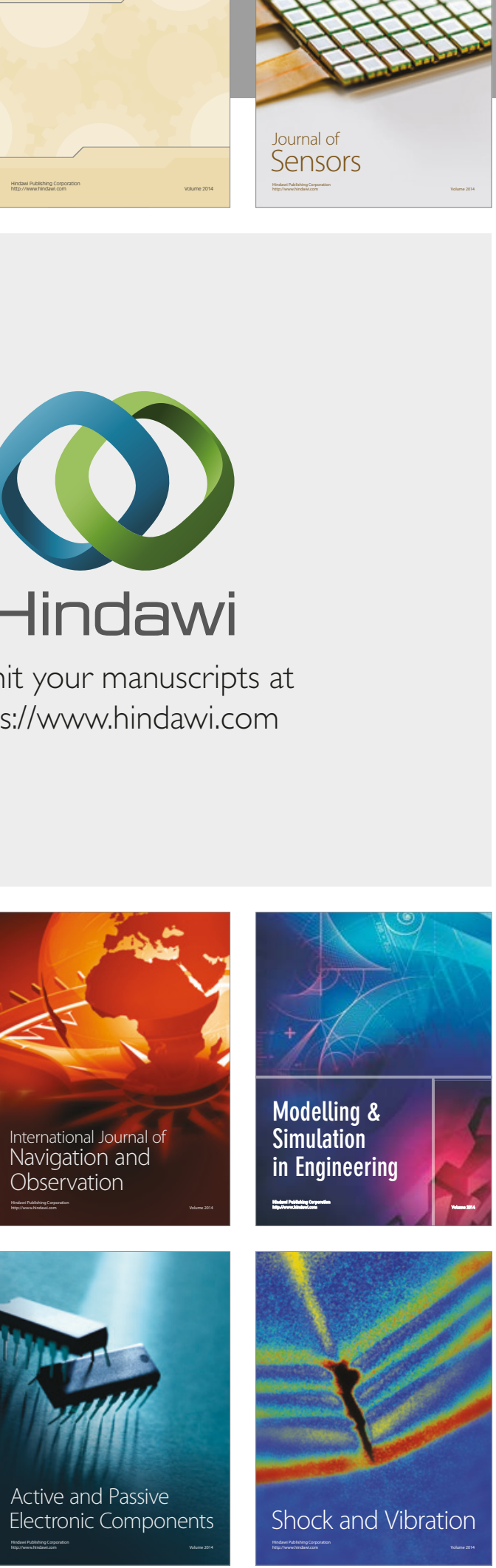
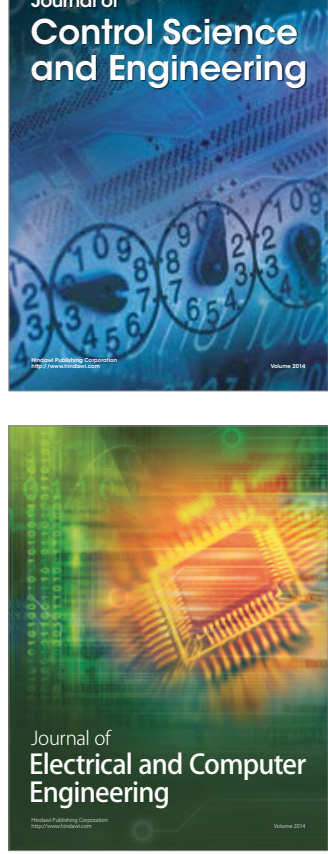

Distributed

Journal of

Control Science

and Engineering
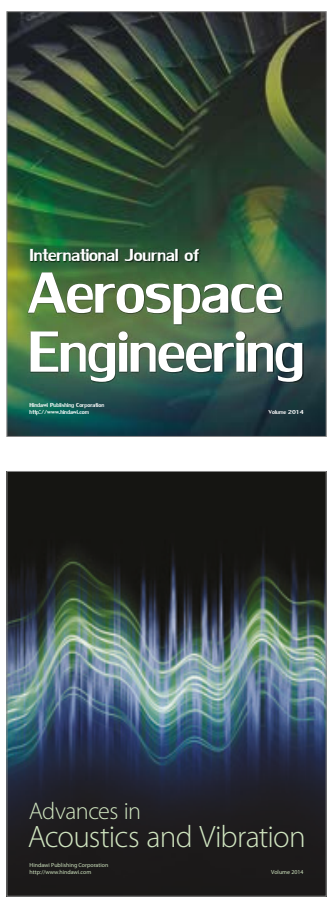

Sensor Networks 